\title{
Using the Mobile Application EDDY for Gathering User Information in the Requirement Analysis
}

\author{
Stephan Hammer, Karin Leichtenstern, Elisabeth André \\ Institute of Computer Science, Augsburg University \\ Universitaetsstr. $6 \mathrm{a}$ \\ 86159 Augsburg, Germany \\ \{hammer, leichtenstern, andre\}@informatik.uni-augsburg.de
}

\begin{abstract}
The users' knowledge and requirements are important factors in the design of products. To ensure the success of products designers and developers have to get to know their target group users better. Over the years a lot of innovative user centered design studies have been written. Many researchers believe that these studies could be used by developers to help them in the process of creating new products. This paper presents a framework called $E D D Y$ that aims at facilitating the development of mobile applications for gathering various kinds of data. Such a framework and applications based on it should be helpful for the data collection during studies such as Cultural Probes and the Experience Sampling Method. These approaches involve the users as the persons who collect the required information themselves. In this paper we evaluate the advantages of EDDY for such studies. We investigated whether there is a significant advantage in using a mobile application for the documentation of a user's everyday life instead of using a classical kit.
\end{abstract}

\section{Author Keywords}

Context-Aware System, Mobile Phones, Requirement Analysis, Cultural Probes, Experience Sampling Method (ESM)

\section{ACM Classification Keywords}

H.5.2 Information Interfaces and Presentation: User Interfaces_Evaluation/methodology, User-Centered Design

\section{General Terms \\ Design, Human Factors}

\section{INTRODUCTION}

The users' knowledge, needs and requirements are the most important factors which decide the success or failure of services and products. For this reason users are mostly consulted to evaluate existing or almost ready products. However detecting and reducing problems at this point of development is time-consuming and expensive. It is therefore es-

This is the author's version of the work. It is posted here for your personal use. Not for redistribution. The definitive Version of Record was published in:

EICS'10, June 19-23, 2010, Berlin, Germany.

Copyright 2010 ACM 978-1-4503-0083-4 sential to include the target user groups early when designing the new product. An important point of user centered design is the requirement analysis. Interesting approaches include the Experience Sampling Method (ESM) [2] and Cultural Probes [5]. They both have the advantage that the users do not need to be observed directly which is time-consuming, limits the number of participants and situations documented by them and also can cause people to act out of character. However, we think that there is still more room to improve the conduction of such self-report studies. For example it is exhausting to carry a traditional Cultural Probes package consisting of a diary, a pen, maybe post-its, cameras or other items for documentation and to sort the gathered data by hand. In comparison to such kits, mobile phones seem to be a better alternative. They are widespread and with all kinds of equipment such as digital cameras or audio recorders in a single device and the possibility of connections via Bluetooth or Internet they are ideal conduits for communication between users and researchers.

We developed a framework called EDDY as a foundation for mobile applications for gathering different kinds of media and data and applicable in private life as well as for studies and evaluations. In this paper we investigate if applications based on EDDY and the automated structuring of gathered data could reduce effort on the part of the user in documenting life and thus increase their motivation for collecting data and for this reason also the amount and quality of the collected data.

Another important part of such studies is the analysis of and reflection on the gathered data. Therefore the study presented in this paper is just the first of two studies that investigate the potential of new technologies for Cultural Probes and the ESM.

The paper is structured as follows: First we present related work which introduces different approaches for requirement analysis and some examples related to the ESM and Cultural Probes and also some systems which support the collecting of different kinds of sensor data in general. Then we give an insight into the framework EDDY and present a user study with an application based on EDDY to document student's everyday life at the university. Finally we discuss the results and give a brief outlook on future work. 


\section{REQUIREMENT ANALYSIS METHODS}

The simplest idea to learn important things about human needs and the situations in which the users could use a later product is by being there. Regarding the development of context-aware technologies Oulasvirta et al. for example introduced an approach called Bodystorming [13] in which the design team observed people of the target group at locations and situations that are identical to or at least similar to those at which the product should be used later. The advantage of Bodystorming is that the developers can focus on important aspects in a real context and do not have to speculate about possible situations. This reduces potential omissions and misconceptions. The main disadvantage of being there, however, is that there are limitations on people observed, places and contexts which is again a risk for missing or misunderstanding needs and requirements.

Experience Sampling Method (ESM) [2] or Cultural Probes [5] at which the researchers are not in situ but confine themselves to interviewing subjects at selected points during the study, deal with this problem. In both approaches the participants receive kits which contain for example a diary or scrapbook to write down important experiences and thoughts, pens, post-its, postcards, cameras or voice recorders. This equipment is available to them for use in documenting their activities or situations that could be of interest for the researchers. ESM and Cultural Probes only differ in the way the users have to conduct their self-report. Participants in the ESM only have to answer several brief questionnaires about their current mood and activities whenever they are requested by alerts delivered via phones or audio players. They do not have to recall situations and feelings after the documentation. On the contrary subjects in Cultural Probes receive only once the instructions, as general as possible, and questions to explain the kind of information required. Then they have to document their activities, situations or feelings spontaneously as these occur or shortly afterwards. After the documentation they have to discuss their reports during a concluding interview. In our opinion this additional effort is acceptable because giving the users the control to decide what to document reduces the risk of missing important information. Also we believe that it could have a negative influence if participants get annoyed by being interrupted by numerous alerts.

Despite these differences both approaches have the advantage that it is possible to receive information about many different users of unfamiliar target groups [6] and private situations in which researchers would never be able to observe subjects such as getting ready for work or having a date. Gaver et al. which introduced the Cultural Probes [6], for example, used traditional packages with elderly people in 3 different countries in Europe to get reports about their lives, their involvement in their cultural environment and their attitude to new technologies. Also some other studies used traditional probe packs to gather information about how families or couples interact by using devices and interactive technologies and what role these technologies play in their relationship ([9], [3]).
Through ease of handling and their role as a permanent companion some approaches also used PDAs or mobile phones like we do to collect data much easier. In Cultural Probes the use of mobile phones could also encourage spontaneous reports, whereas in the ESM they also make it possible to combine alerting, delivering and capturing in one device [2]. As an example for Cultural Probes, Hartnell-Young et al. [15] used mobile phones equipped with cameras to study how students of all ages and also teachers, parents or professionals would use their Lifeblog software. Iversen and Nielsen [8] used a mobile phone camera and audio recording function for collecting digital cultural probes that offered information about the needs and feelings of children in their daily life. Hulkko et al. used Mobile Probes [7] on the one hand to investigate shopping and decision making behavior of buyers and on the other hand to produce new ideas of how to support mobile workers by the development of new products and services. Influenced by the ESM, they also delivered questions via the mobile phone to boost the selfdocumentation. In both studies, similar to our approach, the collected data were sent directly to an online database and automatically sorted by users and questions. Interestingly for our work in the second study Hulkoo et al. [7] used mobile phones with integrated cameras, because the use of non practical and low quality accessory cameras in the first study caused a low number of pictures taken by the users. Similar to MyExperience [4], with EDDY we extend this approach by using the text, photo, video and audio functions of one mobile phone complemented with external sensors for measuring GPS positions and vital signs to receive even more data and to provide a more detailed insight into the user's life.

\section{DATA-COLLECTION FRAMEWORKS}

The projects Affective Diary [11] and the Life Diary [16] were implemented as multimedia diaries for private use with items, such as messages, images, videos and sounds that were collected all over the day and later on were provided via a web interfaces. ContextPhone [14] and ContextWatcher [10] were addressed to ease the synchronous or asynchronous sharing of context data. Additionally ContextWatcher also tried to classify daily habits and criteria for special situations. The most similar mobile application to EDDY is BeTelGeuse [12]. It was used to collect and share several kinds of data. But whereas EDDY was implemented as a framework usable in different fields such as private or sportive documentation and also just like in this paper as a tool used in user studies for several kinds of products, BeTelGeuse was intended for the evaluation of systems and algorithms implemented for recognizing activities and positions.

\section{EDDY - THE FRAMEWORK}

As can be seen in Figure 1 the framework consists of a mobile phone optionally connected with external sensors and a server with an online database in which the gathered data are saved. The mobile phone receives data from the sensors via Bluetooth. The connection between mobile phone and server is built via an HTTP connection. 


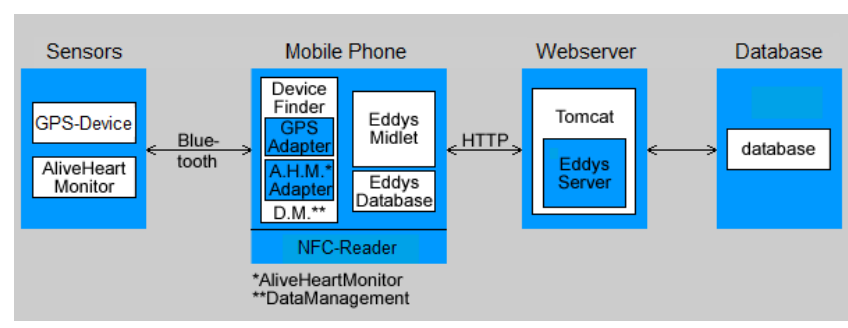

Figure 1. Architecture of Eddy framework

We divided the sources with which we can collect information into two groups. In the first group which we call $a c$ tive sensors we provide all standard functions of the mobile phone: text, video and audio input and taking pictures and optional RFID/NFC. We chose the term active because the user has to actually interact with the device. It is possible to add multiple and different kinds of media to one bundle. The second group is named passive sensors. This group is related to internal and external sensors which gather data continuously. For example, we are using GPS-devices, and the Alive Heart Monitor for measuring vital signs, such as heart rate, ECG and 3D-acceleration [1]. The mobile phone and the external sensors are connected via adapters which are responsible for receiving measured data. The big advantage of this kind of architecture is that new external sensors can easily be integrated by implementing a new adapter and embedding this adapter into the application on the mobile phone. Because of the GPS-device and the Alive Monitor, we have already developed a GPSAdapter and an AliveHeartMonitorAdapter.

The passive sensors can be enabled or disabled by the user who can also set the duration of the measurements and the intervals in which they are conducted. All these settings are saved in the user's profile. When one scan interval is finished the data is automatically sent in the background so that the user is not interrupted at any time.

To reduce the effort of sorting the gathered data, we store the data in diary form with a main title and subtitle supplemented with a time stamp. The time stamp is stored automatically with every data entity gathered whereas the two titles must be entered by the user. The main title must be entered before starting the data collection. All passively gathered data are saved under this title. Before sending a bundle of data collected via active sensors, the user has to enter a subtitle. The media in this bundle are saved under a title consisting of their main title and their subtitle.

If we imagine, for example, that a student in our study documents his/her day at the university the main tile could be University Monday. If he/she wanted to document his/her lunch he/she could generate a bundle of media and enter the subtitle Cafeteria. Afterwards the bundle would be saved under the title University Monday\$Cafeteria as one small collection as part of the major approach University Monday. In our opinion this kind of structure could give rise to convenient advantages for displaying the gathered data for example in web interfaces.

\section{STUDY}

To present the study we now first discuss the background situation to the study, then we describe the realization and in the end we present the results of the evaluation.

\section{Experimental Setting}

In this paper we wanted to investigate EDDY's advantages for gathering data in the field of Cultural Probes and the ESM. Therefore we formulated the following hypotheses:

\section{Hypotheses}

In the following we describe our three hypotheses that we formulated for this evaluation.

- H-1: Quantity of gathered data: The use of a mobile application instead of a traditional Cultural Probes Kit increases the amount of gathered data.

- H-2: Diversity of gathered data: The use of a mobile application instead of a traditional Cultural Probes Kit increases the rate of different kinds of media such as photo, video or audio and combinations of these. For this reason the data gathered then acquire more significance.

- H-3: Attitude during a study: The use of a mobile device facilitates the accumulation of data and thus improves the attitude of the users towards their participation in a lengthy study.

\section{Variables}

To test our hypotheses we set the independent variable $a p$ proach of data collection. Therefore we identified the two values documentation via paper and user-own devices and documentation via EDDY. In the first condition the subjects had to document their day by using paper and pen and optionally also with own devices, such as digital cameras or voice recorders. Also they had to sort and to provide their gathered data by hand. In the second condition each user received a mobile phone on which we had installed EDDY with its functions text input, photo, video and audio recording. All data were saved on an online database.

To eliminate learning effects we used the within-group approach which means that all participants had to document their day with both kinds of documentation. In order to also invalidate all kinds of sequence effects, one half of the participants started with the traditional approach and the other half with EDDY.

Although vital signs or physical activities and especially geographical position could be interesting data, the GPS-device and the Alive Heart Monitor were not used. In this way we maintained the comparability with the usage of paper and user-own devices for which we only could ensure the availability of digital cameras as additional input devices.

Concerning our three hypotheses we defined the three dependent variables: the quantity of stored data, the diversity of stored media and the people's attitude regarding the oneday participation. 
To measure the quantity and diversity of the participants gathered data we analyzed the collected data. To analyze if the person's attitude changed because of the use of EDDY instead of the traditional kits we analyzed the questionnaires which our participants had to fill out after each day of documentation.

\section{Conducting the Study Preliminary to the Study}

First of all each of the subjects had to fill in a short questionnaire with which we assessed the participants' typical usage of their mobile phones. After that we introduced the users to the study scenario and to the documentation approach they had to use first. They were told to document two typical days at the university in a diary form so that we could analyze the gathered data and find some new ideas to support students with new technologies or applications. Because the participants had to use both techniques, they had to come back after the first day of documentation to conduct a short follow-up interview. After this interview they were introduced to the second modality with which they had to continue the documentation. To ensure that the subjects were able to use EDDY, during the introduction all users were encouraged to test the system and to ask questions if necessary. After the second day of report they had to take part in a concluding interview. The related questionnaire also contained questions about the preferred way of documentation and the importance of the different media in particular.

\section{Experiment}

Overall 11 students aged between 20 and 27 took part in our study. The average age of the 4 women and 7 men was 23.1. 9 of the participants studied Computer Science or related fields of study and 2 studied Electrical Engineering. To find out how interested and skilled the students were regarding the use of mobile phones, we asked for the integrated functions in their own mobile phones and if they are using some of them frequently. All mobile phones were enabled to capture photos and videos and most of them $(73 \%)$ were equipped with Bluetooth and and various Internet connections. Additionally, 3 of 11 phones at a time were equipped with GPS or mp3 players respectively radio. However, only 3 students declared use of other functions than calling and short messages very often and 5 declared to use no or few additional functions $(\mathrm{M}=3.64, \mathrm{SD}=2.29)$. The most used functions were taking pictures and calendars (64\%), followed by surfing the web and hearing music $(28 \%)$. Other functions used by the participants were GPS navigation, checking E-Mails and taking videos.

\section{Results}

To evaluate if the results prove or disprove our hypotheses we analyzed the gathered data and the answers in the questionnaires on the basis of dependent variables defined prior to the experiment. These were the quantity of stored data, the diversity of stored media and the people's attitude regarding the one-day participation. All questions provided a rating between 1 meaning not applicable and 7 meaning completely applicable. Furthermore we used a two-sided dependent t-test to detect possible significant results.
For the first hypothesis regarding the quantity of gathered data the results showed no tendency that people collect more data by a mobile application (MA) such as EDDY. Overall, the participants gathered 112 data files by the mobile phone and 103 files by the traditional package. The average was $10.18(\mathrm{SD}=8.18)$ compared to $9.63(\mathrm{SD}=4.76)$. This result could be explained by the highly significant decrease of text messages by using the mobile application (MA: $\mathrm{M}=4.64$, $\mathrm{SD}=3.93$; TA: $\mathrm{M}=8.55, \mathrm{SD}=4.72 ; \mathrm{p}<0.01)$. Some of the participants gave as a reason that they were not used to the T9 text input of the used Nokia 6131 NFC and for that reason they made fewer notes by the mobile phone than by paper and pen. This could be brought out by the also highly significant difference in the amount of words typed in via mobile phone compared to the words written down (MA: M $=28, \mathrm{SD}=25.45$; TA: $\mathrm{M}=74.64, \mathrm{SD}=38.12 ; \mathrm{p}<0.001$ ). Nevertheless some participants stated that mobile phones are faster to pick up than paper and pen. This possibly caused the highly significant increase of taken pictures by EDDY (MA: $\mathrm{M}=5.18, \mathrm{SD}=3.84 ; \mathrm{TA}: \mathrm{M}=0.82, \mathrm{SD}=0.98 ; \mathrm{p}$ $<0.01)$ which resulted in the similar amount of data files in general. Audio files (MA: 5; TA: 0) and video files (MA: 0; TA: 0) were irrelevant.

We already stated that there was a highly significant increase of collected photos and that there were no videos in both approaches and only 5 (MA) respectively 0 (TA) audio files. The result for taking pictures can be explained by some statements of students. One said that photos and videos are more convenient to reflect the documented situations and another stated that the additional digital camera in the cultural probe kit was impractical and therefore he took fewer photos. The results of our analysis mirrored also the results of the ratings received by the media in the concluding questionnaire. In a range from 1 meaning not relevant to 7 meaning highly relevant photography scored an average of $5.73(\mathrm{SD}=1.42)$ whereas capturing video and audio only scored $4(\mathrm{SD}=1.55)$ respectively $3.27(\mathrm{SD}=1.62)$. Text was rated top with a score of $6.45(\mathrm{SD}=0.69)$.

Because combinations of the respective media should be more meaningful than single notes, pictures or other media files, we also compared the collected bundles of data. Whereas $89 \%$ of the bundles stored by the traditional approach only contained single text, just $11 \%$ consisted of text and photos. By contrast the percentage of bundles gathered by the mobile application consisting solely of single text or single pictures only was $19 \%$ respectively $21 \%$. More than half of the bundles (53\%) contained text and photos. The increase of combinations of text and photo by the mobile application also was significant in a one-sided dependent t-test (MA: M $=3, \mathrm{SD}=3.16$; TA: $\mathrm{M}=0.82, \mathrm{SD}=0.98 ; \mathrm{p}<0.05$ ). Packages consisting of text and audio files (2\%), photos and audio files $(3 \%)$ or combinations of more than two kinds of media again were irrelevant.

With these results the second hypothesis regarding the diversity of the gathered data can be rated as proved.

To prove the third hypothesis regarding the participants attitude during a study we analyzed the questions Q1 to Q6 (see 
Fig. 2) asked after each day of self-report. We asked if the documentation with the respective approach was exhausting (Q1), time-consuming (Q2), annoying (Q3), fun (Q4), if the participants would use it again at any time (Q5) or if they would prefer another kind of documentation (Q6).

The results showed that there was a significant advantage of EDDY regarding the question if the type of documentation used was exhausting(Q1) (MA: $\mathrm{M}=1.64, \mathrm{SD}=0.50$; TA: $\mathrm{M}=3.55, \mathrm{SD}=1.92 ; \mathrm{p}<0.05)$. One of the students stated, for example, that at first, documentation by paper and pen was not so exhausting but the subsequent sorting of the gathered notes and pictures was. In a one-sided dependent t-test Q6 also resulted in a significant difference. Whereas only two of the 11 students showed a slight tendency to prefer another approach after using the mobile application, 6 students, which is more than half of the participants, showed a strong preference for the mobile application (MA: $\mathrm{M}=2.82$, $\mathrm{SD}=1.60 ; \mathrm{TA}: \mathrm{M}=4.36, \mathrm{SD}=1.68 ; \mathrm{p}<0.05)$. To underline the results again some statements by the students: Instead of the traditional kit I would prefer an electronic aid that would render post-editing unnecessary.; I would rather use mobile phones than pen and paper. Interestingly all other questions (Q2 - Q5) also showed a tendency in favor of the mobile application (see Fig.2). Therefore, the results indicate that the last hypothesis tend to be proved.

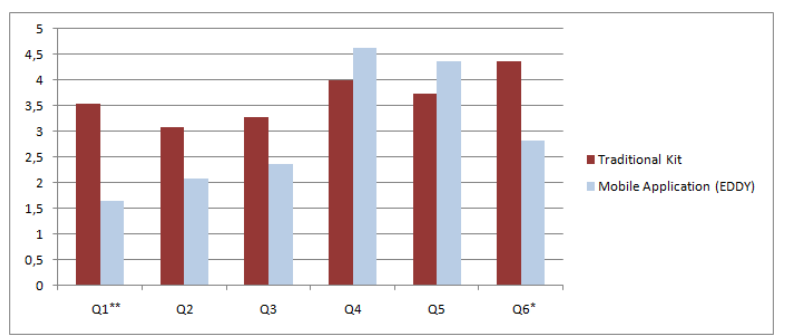

Figure 2. Results of the questionnaire (significant $(p ; 0.05)$ in a *onesided dependent t-test, **two-sided dependent t-test)

\section{DISCUSSION}

In our evaluation conducted with 11 students in a withingroup study we showed tendencies that using EDDY could increase the amount of collected photos and combinations of text and photos. The reason was, for example, the comfort of using a single mobile device instead of a cultural probes kit. One reason why there was no significant increase of the quantity of data in general could be the T9 text input. Some subjects stated that they were not familiar with the text input used by the Nokia 6131 NFC. We offered these mobile phones to hold off the cost occurring by sending the data to the server. It may be that costs can have an influence on the user's attitude. Maybe the amount of text notes collected via the mobile application could be increased in longer lasting studies by some kind of learning effect or by using user-own devices combined with reimbursement or by using mobile phones or PDAs offering a QWERTZ-keyboard or a stylus combined with character recognition. This also could improve the length and quality of notes inputed by EDDY and therefore it could cause a more significant advantage in using a mobile application for the collection of multimedia data. Even though the answers of our question were very balanced (MA: $\mathrm{M}=4, \mathrm{SD}=2.49$; $\mathrm{TA}: \mathrm{M}=3.36, \mathrm{SD}=2.06$ ) whether the subjects felt uncomfortable during some situations, we think that the deficit of video and audio files may be due to the fact that recording videos or audios in public and especially gathering media about unknown people seems still to be unfamiliar. This supposition also was supported by statements of users.

Also we detected that ease of use mobile applications can increase the motivation and stamina of people in self-reporting and therefore we think that our outcome mentioned before will be intensified by conducting the documentation over a longer period.

However, we want to clarify that using either mobile applications or traditional kits depends as well on target groups and user needs as the later service or product. As our results showed, using technology in studies with experienced users could be very helpful. However, conducting studies with, for example, elderly people requires familiar and traditional techniques to not ask too much of them [6].

\section{CONCLUSIONS}

In this paper we introduced some systems for the collection of different kinds of information about the user and his/her everyday life. Such systems are helpful both in private and in studies and evaluations. For example, in the EU project METABO information about the metabolism and lifestyle of diabetics is used to improve the treatment of their disease. Our framework EDDY was developed to extend the technical possibilities of earlier work and to facilitate the development of context aware mobile applications applicable in both fields. EDDY covers the functions text, photo, video and audio and also the collection of measurements via external sensors in a single mobile application. Also it relieves the user's burden of sorting gathered data by hand. In this work we focused on the advantages EDDY and applications based on it could offer for the data collection in user centered design processes, such as Cultural Probes or the Experience Sampling Method (ESM). We prefer these approaches because they overcome the restrictions on observed people and situations caused, for example, by approaches like Bodystorming. To validate possible advantages of a mobile application in comparison to the traditional approach we compared them in a user study. The results showed that there was no difference in the quantity of gathered data, but there was a tendency of an increase of the stored data's diversity and the users' attitude during the study.

In future we want to add the data delivered by our external GPS-device and the Alive Heart Monitor to get more meaningful information about the user's context in which he/she collected the data. Also we are planning to conduct our second study regarding the use of Cultural Probes and the ESM focusing on the analysis of the gathered data in the 
design phase. Therefore we are currently developing web interfaces with which we can display the temporal and geographical connections between single data collected by the users. Because this study will be conducted with designers and developers we also will receive experts' ratings of the quality of the gathered data in the first study.

\section{ACKNOWLEDGEMENTS}

The work described in this paper is funded by the EU under research grant ICT-216270-METABO.

\section{REFERENCES}

1. Alive technologies. alive wireless heart monitor. product highlights and specification.

2. S. Consolvo and M. Walker. Using the experience sampling method to evaluate ubicomp applications. IEEE Pervasive Computing, 2(2):24-31, 2003.

3. T. Dalsgaard, M. B. Skov, M. Stougaard, and B. Thomassen. Mediated intimacy in families: understanding the relation between children and parents. In IDC '06: Proceedings of the 2006 conference on Interaction design and children, pages 145-152, New York, NY, USA, 2006. ACM.

4. J. Froehlich, M. Y. Chen, S. Consolvo, B. Harrison, and J. A. Landay. Myexperience: a system for in situ tracing and capturing of user feedback on mobile phones. In MobiSys '07: Proceedings of the 5th international conference on Mobile systems, applications and services, pages 57-70, New York, NY, USA, 2007. ACM.

5. G. Gaffney. What is cultural probes? http : / / www . infodesign. com . au, 2006.

6. B. Gaver, T. Dunne, and E. Pacenti. Design: Cultural probes. interactions, 6(1):21-29, 1999.

7. S. Hulkko, T. Mattelmäki, K. Virtanen, and T. Keinonen. Mobile probes. In NordiCHI '04: Proceedings of the third Nordic conference on Human-computer interaction, pages 43-51, New York, NY, USA, 2004. ACM.

8. O. S. Iversen and C. Nielsen. Using digital cultural probes in design with children. In IDC '03: Proceedings of the 2003 conference on Interaction design and children, pages 154-154, New York, NY, USA, 2003. ACM.
9. J. Kjeldskov, M. Gibbs, F. Vetere, S. Howard, S. Pedell, $\mathrm{K}$. Mecoles, and M. Bunyan. Using cultural probes to explore mediated intimacy. Australasian Journal of Information Systems, 11(2), 2007.

10. J. Koolwaaij, A. Tarlano, M. Luther, P. Nurmi, B. Mrohs, A. Battestini, and R. Vaidya. Context watcher: Sharing context information in everyday life. In in Proceedings of the IASTED conference on Web Technologies, Applications and Services (WTAS). IASTED, pages 12-21, 2006.

11. M. Lindström, A. Ståhl, K. Höök, P. Sundström, J. Laaksolathi, M. Combetto, A. Taylor, and R. Bresin. Affective diary: designing for bodily expressiveness and self-reflection. In $\mathrm{CHI}$ '06: $\mathrm{CHI}$ '06 extended abstracts on Human factors in computing systems, pages 1037-1042, New York, NY, USA, 2006. ACM.

12. P. Nurmi, J. Kukkonen, E. Lagerspetz, J. Suomela, and P. Floréen. Betelgeuse: a tool for bluetooth data gathering. In BodyNets '07: Proceedings of the ICST 2nd international conference on Body area networks, pages 1-8, ICST, Brussels, Belgium, Belgium, 2007. ICST (Institute for Computer Sciences, Social-Informatics and Telecommunications Engineering).

13. A. Oulasvirta, E. Kurvinen, and T. Kankainen. Understanding contexts by being there: case studies in bodystorming. Personal Ubiquitous Comput., 7(2):125-134, 2003.

14. M. Raento, A. Oulasvirta, R. Petit, and H. Toivonen. Contextphone: A prototyping platform for context-aware mobile applications. IEEE Pervasive Computing, 4(2):51-59, 2005.

15. E. H. Young and F. Vetere. Lifeblog: A new concept in mobile learning? In WMTE '05: Proceedings of the IEEE International Workshop on Wireless and Mobile Technologies in Education, pages 174-178, Washington, DC, USA, 2005. IEEE Computer Society.

16. R. Youngho, S. Jennifer, K. Junghun, and C. Amy. Create, sync, share: the role of mobile device on social entity. In MobileHCI '07: Proceedings of the 9th international conference on Human computer interaction with mobile devices and services, pages 452-456, New York, NY, USA, 2007. ACM. 